\title{
PREVALENCIA DE SÍNDROME DE BURNOUT EN LOS ESTUDIANTES DE LA CARRERA DE ENFERMERÍA DE LA UNIVERSIDAD CENTRAL DEL ECUADOR
}

\section{THE PREVALENCE OF THE BURNOUT SYNDROME AMONG STUDENTS FROM THIRD TO EIGHTH SEMESTER WHO ARE MAJORING NURSING AT CENTRAL UNIVERSITY OF ECUADOR}

\author{
Luzmila de Jesús Carvajal Andrade ${ }^{1}$ \\ Belén del Rocío Logacho Villacís ${ }^{2}$ \\ Ramiro Rogelio Rojas Jaramillo ${ }^{3}$
}

Recibido: 2019-07-26 / Revisado: 2019-10-16 / Aceptado: 2019-10-25 / Publicado: 2020-01-01

Forma sugerida de citar: Carvajal-Andrade, L., Logacho-Villacís, B. y Rojas-Jaramillo, R. R. (2020). Prevalencia de síndrome de burnout en los estudiantes de la carrera de enfermería de la Universidad Central del Ecuador. Retos de la Ciencia. 4(8), pp. 38-54. https://doi.org/10.53877/rc.4.8.20200101.04

\section{RESUMEN}

El propósito de este estudio es determinar la prevalencia de síndrome de burnout en los estudiantes de tercero a octavo semestre de la Carrera de Enfermería; es un estudio de prevalencia, en el cual, se utilizaron la encuesta con su respectivo cuestionario para la recolección de datos. El cuestionario estuvo dividido en 5 secciones y fue aplicado a 172 estudiantes. La información recogida fue analizada calculando la media y la desviación estándar para el burnout académico; mientras que, para el burnout laboral, se utilizó la puntuación de las escalas alto, medio y bajo. Los resultados demuestran que la prevalencia del síndrome de burnout académico y laboral fue baja. El 2,3\% de estudiantes tuvieron síndrome de burnout académico (Intervalo de confianza: 95\%, límite inferior: 0.44\% y límite superior: $4.21 \%$ ) con una probabilidad de $3.52 \%$, mientras que el síndrome de burnout laboral fue del $4 \%$ en los estudiantes del Internado Rotativo, con una probabilidad de 1,22\%.

Palabras clave: síndrome de burnout, estrés, enfermería.

\footnotetext{
1 Enfermera, servidor público 2, Ministerio de Salud Pública. Ecuador. E-mail: luzmilacarvajalcomputronic@gmail.com

2 Enfermera, servidor público 2, Ministerio de Salud Pública. Ecuador. E-mail: belen4294@gmail.com

${ }^{3}$ Magister en Ciencias, Docente en la Facultad de Ciencias Médicas de la Universidad Central del Ecuador. Ecuador. E-mail: rrrojasj@uce.edu.ec
} 


\section{ABSTRACT}

This research has been conducted in order to determine the Prevalence of the burnout syndrome among students from third to eighth semester who are attending the Nursing School. It was a prevalence study; the data were collected using the survey's technique, in a questionnaire divided in five sections applied to 172 students. The information analysis was calculated using the Mean method and the Standard Deviation for the Academic burnout, while for the Labor burnout it was utilized the punctuation of: high, medium and low scales. The outcome results showed that the prevalence of the burnout Syndrome in both academic and labor was low. The $2.3 \%$ of students had the Academic burnout, (Confidence interval: 95\%, lower limit: 0.44\% and upper limit: $4.21 \%$ ) with a probability of $3.52 \%$, on the other hand the Labor burnout was of $4 \%$ among students in the shifting internship, with a probability of $1.22 \%$.

Key words: Syndrome burnout, Stress, nursing.

\section{INTRODUCCIÓN}

En el trayecto de la historia, los seres humanos han buscado adaptarse al medio en el que se ven inmersos, satisfaciendo asi sus necesidades, sin embargo, diversas circunstancias les puede ocasionar tensión, la cual, si no es manejada adecuadamente, se vuelve estrés. El estrés crónico, es el resultado de demandas y presiones durante periodos de tiempo prolongados. Este tipo de estrés tiene gran importancia en el proceso adaptativo, ya que está directamente relacionado con la salud y la enfermedad, pudiendo ocasionar alteraciones físicas, neuropsiquiátricas (ansiedad y depresión), trastornos metabólicos (obesidad, diabetes mellitus tipo 2), cardiovasculares (hipertensión, infarto de miocardio) y del sueño (insomnio). (Moscoso, 2014, p. 62).

Por otro lado, en el año 1974 aproximadamente, Hans Selye utilizó los términos "Eustrés y distrés". El primero es catalogado como positivo, convirtiéndose en un estímulo para afrontar una situación que implica salir de la zona de confort, considerando la necesidad de niveles moderados de estrés, para responder satisfactoriamente ante las exigencias propias y del entorno, evitando el trabajo mediocre (Neave, 2016, p. 10). El distrés, se relaciona con la respuesta inadecuada a ciertos estresores, causando así un desbalance en la formación de emociones positivas y negativas, sensaciones de tristeza, inseguridad, confusión y preocupación, hasta la experiencia de síntomas más severos como ansiedad, depresión, ira, aislamiento social y pérdida de esperanza (Moscoso, 2014, p. 59).

El síndrome de burnout es una secuela del estrés crónico, desencadena progresivamente agotamiento físico o mental, falta de motivación y cambios comportamentales (Martínez y Caballero, 2016, p. 2). Ha sido investigado en diferentes poblaciones, relacionándose con mayor frecuencia en el ámbito laboral, sin embargo, actualmente hay diferentes aspectos evaluados, uno de ellos es el entorno académico.

Históricamente, el síndrome de burnout laboral ha ido en aumento, es así que, en el año 1980 Kyriacou determinó su incidencia del 25\% en los profesores, en 1982 Maslach y Jackson establecieron un 20 a 35\% en los profesionales de enfermería (Flores, 2016, p. 37). A nivel mundial, se determinó que 2 países tienen un alto grado de síndrome de burnout en el ámbito laboral, encontrándose a México como el de 
mayor prevalencia con un $75 \%$, seguido de China $73 \%$, estos datos fueron entregados por la Organización Mundial de la Salud en el 2017 (FORBES, 2017, p. 1). A escala nacional, la prevalencia en los profesionales sanitarios es de aproximadamente $30 \%$ (Veletanga, 2017, p. 1).

Algunos de los factores asociados al síndrome de burnout son, por ejemplo: carga horaria excesiva, áreas de atención a pacientes en estado crítico, malas relaciones interpersonales, determinantes sociodemográficos, mal uso de estrategias de afrontamiento, preocupación deficiente o excesiva por la solución de problemas, clima organizacional impropio, entre otros (Rosales, 2014, p. 9-11) (Torres, 2017, págs. 910; Ramírez, 2017, p. 2).

Desde el enfoque académico, durante la carrera universitaria el individuo adquiere conocimientos, acorde a su perfil profesional y desarrolla nuevas habilidades y destrezas, lo cual podría generar un ambiente de estrés basado en la exigencia de la institución, algunos logran implementar estrategias adecuadas para sobrellevar este entorno, pero también hay quienes no consiguen hacerlo. (Ticona, Paucar, y Llerena, 2010, p. 1-2). Por otro lado, el síndrome de burnout en estudiantes universitarios se caracteriza por la exposición de manera prolongada a estresores crónicos, que incluyen los contextos tanto organizacional-académico (competitividad, presiones y sobrecargas) como familiar, social y económico, por lo que pueden desarrollar actitudes negativas, dificultad en el desempeño estudiantil y repercutir así en la salud (Marenco, Suarez, y Palacio, 2017, p. 45).

En la ciudad de Barranquilla, se encontró una incidencia del síndrome de burnout de 10,67\% en universitarios. (Estrada, De La Cruz, Bahamón, Perez, y Caceres, 2017, p. 9). En un estudio realizado en Internos Rotativos de Medicina, se determinó que hay altos niveles de tiene cansancio emocional (74\%) y de despersonalización (64\%), se concluyó que esto ocurre debido a la sobrecarga horaria (Fuera de lo acordado en el contrato, debido a la demanda abundante de pacientes) (Flores, 2016, p. 52). En los estudiantes de enfermería de la segunda especialidad, en Centro Quirúrgico de la Universidad Nacional Mayor de San Marcos-Lima, el 52\% presentó un nivel medio de agotamiento emocional y $40 \%$ niveles medios de despersonalización, sin embargo, el $72 \%$ tuvo un nivel medio de realización personal (Norabuena, 2016, p. 49).

Las manifestaciones del síndrome de burnout, aumentan a medida que avanza el ciclo académico o el periodo laboral, dentro de esto se encuentra el agotamiento, despersonalización, baja realización personal, ineficacia, cinismo, actitudes negativas, desvalorización, pérdida del interés frente al estudio o trabajo, disminución del rendimiento y por ende bajos resultados en evaluaciones cognitivas, con un mayor riesgo de deserción académica, la cual a su vez se asocia con una inadecuada orientación educativa y profesional al elegir la carrera (Norabuena, 2016, p. 25,26) (Ticona et al, 2010, p. 2)

En lo que concierne a los principales tipos de instrumentos, para evaluar la presencia o no del síndrome de burnout, incluyen el "Maslach burnout InventoryGeneral Survey (MBI-GS)", establecido por Schaufeli, Leiter, Maslach y Jackson, en el año 1996, evalúa a todo tipo de profesionales, cuyas dimensiones son: agotamiento, cinismo e ineficacia profesional, y el "Maslach burnout Inventory-Student Survey (MBI-SS)", implementado por Schaufeli, Salanova, González-Romá y Bakker en el 2002, con un enfoque netamente hacia los estudiantes universitarios, evalúa los mismos parámetros que el anterior. Bresó, Salanova, Schaufeli, Equipo de investigación, y Nogareda, 2013, p. 5). 
Existen estrategias de prevención que consisten en realizar un esfuerzo por el autocontrol al estrés, adaptación a la nueva realidad, búsqueda y resolución del problema, descanso suficiente, mantener salud mental adecuada, decidir si la situación estresante va a dejar frustración o, al contrario, si será pasajera y constructiva. En el área sanitaria, es importante trabajar con empatía y ética profesional, evitando implicarse emocional y personalmente de forma absoluta con el paciente, pero se lo puede hacer de forma parcial. (Aguas, Vargas, Guzman, y Ferrer, 2017, p. 16) (Díaz, 2017, p. 41-42).

El sistema de educación universitaria en el Ecuador, está atravesando por un proceso de evaluación, acreditación y categorización de carreras universitarias y escuelas, ejecutada por el Consejo de Aseguramiento de la Calidad de la Educación Superior (CACES), que busca que los procesos educativos que se brindan se desplieguen con alta calidad, para obtener grandes resultados en el desarrollo del país, por lo cual, la Carrera de Enfermería forma profesionales, a través de distintas metodologías utilizadas en el proceso educativo, para cumplir así con su misión y visión. Desde este enfoque, adicionalmente a la formación en el contenido teórico, las prácticas se llevan a cabo en los diferentes establecimientos de salud, previamente coordinados con las autoridades de las Unidades Asistenciales Docentes (UAD), siendo guiadas por docentes de alto nivel profesional. Para cumplir con los objetivos de aprendizaje, los estudiantes deben efectuar trabajos autónomos (Individuales o grupales) y actividades académicas extrahospitalarias.

Hay un porcentaje de universitarios que se desplazan desde otras provincias hacia su lugar de estudio, por lo que muchos de ellos se ven obligados a vivir solos o con personas de lazos de consanguinidad lejanos (Primos, amigos, y demás), lo que complica su nivel de adaptación al nuevo entorno, mientras que otros; tienen relaciones interpersonales inadecuadas (A nivel docente o con sus pares), no disponen de recursos económicos necesarios o no cuentan con el apoyo familiar suficiente. En el programa del Internado Rotativo, al ser una etapa de afirmación profesional, los estudiantes tienen mayor relación laboral que académica, en comparación con los semestres anteriores, es decir, según la norma, deben cumplir 120 horas laborales y 40 horas académicas. Todo esto, en conjunto, repercute en el cumplimiento de actividades y necesidades diarias básicas, por lo que se pueden presentar problemas tales como: alimentación inadecuada, difícil acceso a la atención de salud, menos disponibilidad de tiempo para descansar, distanciamiento familiar, entre otras.

Acorde a los argumentos expuestos anteriormente, se originan múltiples tipos de estresores internos y externos, que requieren un afrontamiento adecuado para que no se convierta en un ente patológico, como se ha observado en ciertos estudiantes que afrontan de manera incorrecta estas situaciones, generando en mayor proporción, hábitos poco saludables como el consumo de alcohol y otras sustancias psicotrópicas.

Probablemente estos problemas interfieran en el logro de los objetivos de aprendizaje, originando dudas acerca de la elección de su carrera profesional y/o deserción académica, mientras que quienes realizan prácticas tienen mayor riesgo de ofrecer una calidad de atención deficiente al paciente de cuidado directo, además, podría influir en el desarrollo del síndrome de burnout.

De acuerdo a los argumentos planteados, se estudió la prevalencia del síndrome de burnout académico, en el grupo poblacional de estudiantes de tercero a octavo semestre de la Carrera de Enfermería, y la prevalencia del síndrome de burnout 
laboral en los estudiantes del Internado Rotativo, considerando que este problema se encuentra relacionado con ciertas características asociadas a las condiciones de vida, problemas socio-económicos, entre otros, que los predispone al bajo rendimiento, por lo cual también se identificó factores $s$ y manifestaciones clínicas asociadas al síndrome de burnout.

\section{MÉTODOS}

Estudio de prevalencia, realizado con una muestra aleatoria de 172 estudiantes universitarios, divididos porcentualmente de tercer a octavo semestre de la Carrera de Enfermería de la Universidad Central del Ecuador del periodo académico abril 2018-marzo 2019. La prevalencia del síndrome de burnout del $80 \%$, se consideró en base al promedio aproximado según la OMS en 3 países a nivel mundial, para el sector universitario del área de salud (FORBES, 2017, p. 1) (Veletanga, 2017, p. 1). Se trabajó con esta prevalencia puesto que hay más estudios realizados en el ámbito laboral en comparación con el académico, que en realidad son muy escasos en estudiantes de la Carrera de Enfermería.

Se utilizó la técnica de la encuesta y como instrumento de recolección de datos, un cuestionario, dividido en 5 secciones acordes a cada una de las variables que se midieron en la investigación (Datos socio-demográficos; factores asociados; ítems que valoran tanto la prevalencia del burnout académico según criterios del MBI-SS, como para determinar la prevalencia del burnout laboral, basado en MBI-GS, modificados de acuerdo a la realidad de esta investigación; principales manifestaciones asociadas al síndrome de burnout).

Se elaboró el cuestionario y se realizó la respectiva validación cualitativa, de contenido, criterio y constructo. La fiabilidad y validez, se comprobó con el coeficiente alfa de Cronbach, para lo cual se aplicó una prueba piloto al $14,45 \%$ de la muestra total. En el análisis de correlación se estableció que ocho elementos (Apoyo familiar, familia está de acuerdo con la carrera escogida, oferta laboral de la profesión, recursos económicos suficientes, planificación adecuada del tiempo, motivación en conseguir objetivos, influencia positiva en la vida de las personas y asistencia normal a clases y/o prácticas), correlacionaron de forma negativa, obteniendo una puntuación de 0.885 (Bueno), posteriormente dichos elementos fueron eliminados y se obtuvo el 0.91 (Excelente) que indicó que el instrumento es fiable y valido, por lo tanto, pudo ser aplicado para determinar la prevalencia de síndrome de burnout en los estudiantes de la Carrera de Enfermería de la Universidad Central del Ecuador.

La realización de este trabajo fue aprobada por el comité de Bioética de la Facultad de Ciencias Médicas de la Universidad Central del Ecuador, en concordancia a lo normado por la declaración de Helsinski.

\section{Análisis estadístico}

Se determinó la prevalencia del síndrome de burnout académico en los estudiantes de tercero a octavo semestre, calculando la suma, media y promedio en las subescalas y se definió un caso positivo considerado tres criterios, es decir, valores superiores al promedio más la desviación estándar en cansancio $(\geq 3,8)$ y cinismo $(\geq 2,3)$, pero valores inferiores al promedio menos la desviación estándar de autoeficacia académica $(\leq 2,6)$ (Bolaños y Rodriguez, 2015, p. 47). Se determinó la prevalencia del síndrome de burnout laboral en los estudiantes del Internado Rotativo, 
si uno de los Internos obtuvo altas puntuaciones en cansancio emocional (8-15) y despersonalización (6-15), pero bajas en realización personal (1-5). (Jiménez y García, 2016, p. 119). Se calculó la prevalencia con intervalos de confianza (IC); límite superior (LS) y límite inferior (LI), para un nivel de significación del 95\% y un error del $5 \%$, sin embargo, considerando que la prevalencia fue un porcentaje cercano a 0 y en el caso del síndrome de burnout laboral, los intervalos encontrados fueron muy amplios, denotaron una escasa precisión; por lo que el cálculo de la probabilidad, se hizo a través de la ley binomial (Mendizábal, Noboa, Cueva y Buitrón, 2017, p. 67).

En primera instancia, se utilizó el análisis factorial como una técnica de reducción de datos, para identificar los principales factores y manifestaciones asociadas al síndrome de burnout. En la matriz de componente rotado, se obtuvieron 7 y 8 componentes respectivamente, sin valores negativos en las variables. Sin embargo, estos componentes mostraron variables que no se pudieron asociar, por lo que se analizaron los datos, según el orden de los objetivos. Por otra parte, en las manifestaciones físicas, emocionales y conductuales, se tabuló creando una variable binaria, para analizarlas mediante gráficos de barras.

Se realizaron tablas de contingencia, para analizar la relación entre factores sociodemográficos, personales, académicos y del contexto socio económico VS Prevalencia del síndrome de burnout académico en estudiantes de tercero a octavo y también entre los factores de tipo laboral y el síndrome de burnout laboral, en estudiantes del Internado Rotativo. Se utilizaron pruebas de chi-cuadrado con corrección de Yates, para analizar si existían relaciones estadísticamente significativas entre las variables en análisis, luego se complementó con el cálculo de los coeficientes de contingencia para analizar la dirección y fuerza de la relación estadística. Para el análisis de los datos se utilizó el programa IBM SPSS Stadistics (versión 25 ) con un $100 \%$ de los datos analizados válidos.

\section{RESULTADOS}

La prevalencia del síndrome de burnout académico fue de 2,3\% (IC de 95\%, con LI de $0.44 \%$ y LS de $4.21 \%$ ) con una probabilidad de 3,52\%. De manera general, en las subescalas se encontraron valores altos de agotamiento $(19,2 \%)$, cinismo $(18,6 \%)$ y $(29,7 \%)$ con bajos niveles de autoeficacia académica. Agrupado por semestres, se hallaron niveles altos de agotamiento, de ellos predomina en tercero (7\%); y se repite en tercero y sexto, para niveles altos de cinismo $(4,7 \%)$, y niveles bajos de autoeficacia académica (5,8\%). En los alumnos del Internado Rotativo, la prevalencia del síndrome de burnout laboral fue de 4\% con una probabilidad de 1,22\%. En cuanto a las subescalas, de forma agrupada por semestres, predominó séptimo con niveles altos de: cansancio emocional (30\%); despersonalización (10\%) y con niveles bajos de realización personal (12\%).

Factores asociados al síndrome de burnout académico, en estudiantes de tercer a octavo semestre.

\section{Características sociodemográficas}

El promedio de edad fue de $23 \pm 2,67$ años, con una mediana y moda de 22 , edad mínima de 19 y máxima de 36. La mayoría de los estudiantes corresponden al sexo femenino $(84,9 \%)$ de las cuales $(2,3 \%)$ tienen síndrome de burnout académico y pertenecen a tercer semestre, sexto y octavo. La mayor parte de la muestra 
corresponde al estado civil soltera/o (85,5\%) y de ellos (2,3\%) tienen síndrome de burnout académico, mientras que $(19,2 \%)$ viven solos o con familiares con lazos de consanguinidad lejanos. Finalmente $(35,5 \%)$ señalaron que tienen cargas familiares.

\section{Factores personales}

Del total de la muestra el $68 \%$ de estudiantes manifestó dormir menos de 6 horas al día; el $60 \%$ indicó que a veces han tenido relaciones tensas con sus compañeros del aula; un $43 \%$ con sus docentes, el $60 \%$ con sus compañeros del aula, el $45 \%$ con pacientes o sus familiares y el $61 \%$ señaló que a veces algunos médicos, enfermeras/os u otro personal de salud se ha dirigido en tono autoritario hacia él/ella; el $56 \%$ refirió que nunca realizó actividades de ocio y/o relajación; el $48 \%$ ha mencionado que tan solo a veces puede controlar adecuadamente situaciones estresantes. Es importante señalar que, en cada variable, dentro de los resultados mencionados el 1,7\% tiene síndrome de burnout académico.

\section{Factores de tipo académico}

De la muestra encuestada, más del $90 \%$ de alumnos manifestó que si recibe tutorías académicas tanto en clases como en práctica; el $60 \%$ refirió que nunca ha tenido acceso a la asociación estudiantil de la Carrera; un $59 \%$ señaló que esta carrera no fue la primera opción durante la postulación en el Sistema de Educación Superior utilizado en el momento de su ingreso; el $41 \%$ indicó que tuvo dificultad en el acceso a bibliotecas físicas y/o virtuales de la carrera; el 35\% indicó que siempre existe competitividad entre compañeros de clases; el $28 \%$, señaló que a veces la metodología de enseñanza es adecuada en la teoría-práctica. Entre los resultados positivos, se encontró que el $40 \%$ casi siempre domina las habilidades y destrezas necesarias; el 38\%, manifestó que casi siempre hay satisfacción con las temáticas abordadas y el $47 \%$ expresó que siempre cuenta con la presencia de un docente en actividades teóricas. Dentro de cada grupo mencionado el 1,7\% tiene síndrome de burnout académico.

El 45\%, refirió que cuentan con supervisión continua en la práctica por tutores y/o profesionales del área. De este grupo, el 1,2\%\% tiene síndrome de burnout académico.

Por otro lado, el $85 \%$ de estudiantes expresó que prefiere realizar los trabajos de manera individual; el $78 \%$ enunció nunca haber recibido algún incentivo o beca económica por excelencia académica; un $43 \%$ dijo que casi siempre o siempre tienen temor y ansiedad durante las evaluaciones académicas. De los resultados mencionados el 2,3\% tiene síndrome de burnout académico.

\section{Factores del contexto socioeconómico}

El $76 \%$ de la muestra encuestada señaló que por lo general tan solo a veces o en otros casos nunca tienen deudas económicas, sin embargo, dentro de éste grupo está el 2,3\% de estudiantes que tienen síndrome de burnout académico.

Factores asociados al síndrome de burnout laboral (Estudiantes del Internado Rotativo).

El $42 \%$ de Internos Rotativos de Enfermería refirieron realizar horas en exceso; el $64 \%$ indicó que a veces algunos médicos, enfermeras/os u otro personal de salud se ha dirigido hacia ellos en tono autoritario. En cuanto al contacto con el dolor y riesgo inminente de mortalidad, el $52 \%$ señaló que a veces ha vivido este tipo de 
experiencia; finalmente el $50 \%$ de estudiantes dijo que a veces manejaron pacientes en estado crítico o de alta complejidad. Cabe señalar que dentro de los resultados mencionados se encuentra un porcentaje variable de los estudiantes que si tienen el síndrome de burnout laboral.

\section{Manifestaciones asociadas al síndrome de burnout}

En cuanto a las manifestaciones físicas, el gráfico 1 demuestra que más del $50 \%$ de estudiantes de la Carrera de Enfermería han presentado al menos una vez nausea, palpitaciones, gastritis, gripe frecuente, rigidez muscular, dolor de cabeza, cuello y espalda. Mientras que crisis asmáticas, alteraciones inmunológicas (alergias, enfermedades autoinmunes, u otras), diarrea, dolor precordial y migraña, en un porcentaje menor al $50 \%$.

Figura 1: Manifestaciones físicas asociadas al síndrome de burnout

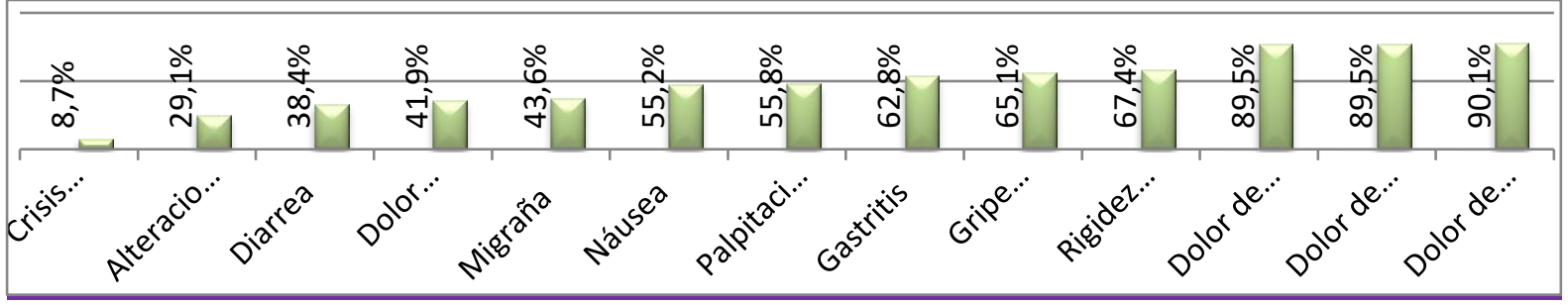

Fuente: Instrumento de recolección de datos.

Elaborado por: Elaboración propia

En lo que se refiere a las manifestaciones emocionales, en la figura 2, se observa que $89 \%$ de estudiantes, refirieron que han tenido dificultad para la concentración, seguido del $82,6 \%$, que han tenido cambios bruscos de humor.

Figura 2: Manifestaciones emocionales asociadas al síndrome de burnout

\begin{tabular}{rl|} 
Dificultad para la concentración & $89,0 \%$ \\
Cambios bruscos de humor & $82,6 \%$ \\
Ansiedad & $79,1 \%$ \\
Soledad & $64,5 \%$ \\
Baja autoestima & $55,2 \%$
\end{tabular}

Fuente: Instrumento de recolección de datos.

Elaborado por: Elaboración propia

Las manifestaciones conductuales señalan en la figura 3, que un alto porcentaje de encuestados, es decir $59,9 \%$ de alumnos consumen alcohol, seguido del $55,8 \%$ que han pensado en abandonar la carrera.

Figura 3: Manifestaciones conductuales asociadas al síndrome de burnout

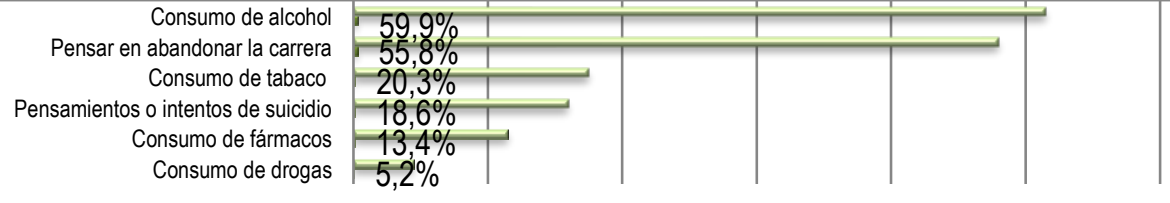

Fuente: Instrumento de recolección de datos.

Elaborado por: Elaboración propia. 
Cálculo de chi cuadrado (X2) con corrección de Yates (p 0,00) de los resultados más relevantes, entre factores y manifestaciones asociadas al síndrome de burnout.

De los encuestados, el $48 \%$ de estudiantes no pueden controlar de manera adecuada situaciones de estrés, los mismos que a su vez, presentan ansiedad. La relación de esta variable es con un valor de $p$ de 0,00 y un coeficiente de contingencia de 0,27 que indica una dirección y fuerza de la relación estadística baja.

Por otro lado, el $34,9 \%$ de encuestados que no pueden controlar situaciones estresantes, tienen a la vez baja autoestima. La relación de esta variable es con un valor de $p$ de 0,00 y un coeficiente de contingencia de 0,27 que indica una dirección y fuerza de la relación estadística baja. También un 46,5\% de universitarios que sienten temor durante sus evaluaciones académicas, tienen a la vez sentimientos de soledad. La relación de esta variable es con un valor de $p$ de 0,00 y un coeficiente de contingencia de 0,29 que indica una dirección y fuerza de la relación estadística baja.

Finalmente, el $46,5 \%$ de alumnos que no tienen actividades de ocio y/o relajación tienen baja autoestima. La relación de esta variable es con un valor de p de 0,00 y un coeficiente de contingencia de 0,28 que indica una dirección y fuerza de la relación estadística baja.

\section{DISCUSIÓN}

Los resultados encontrados en el presente estudio hacen una aproximación sobre la problemática en relación con el síndrome de burnout tanto académico como laboral.

Según la tabulación de los datos obtenidos, se concluyó que, del total de la muestra estudiada, existe una baja prevalencia, el 2,3\% de alumnos tienen síndrome de burnout académico (Estudiantes de tercero, sexto y octavo semestre), con IC $0.44 \%$ (LI) y $4.21 \%$ (LS), con una probabilidad de 3,52\%. Estos resultados son similares con un estudio realizado por (Bolaños y Rodriguez, 2015, p. 1), quienes determinaron un burnout académico del 18,8\%, de los cuales el $29,1 \%$ pertenecía a quinto semestre. Sin embargo, esta prevalencia es muy baja en comparación con los resultados obtenidos en una investigación denominada "burnout en estudiantes de enfermería de una universidad privada", donde el $100 \%$ de los alumnos tuvieron burnout académico (Uribe y lllesca, 2016, p. 1).

La prevalencia del estudio actual está conformada por estudiantes que tienen mayor demanda académica, porque en su respectivo proceso de aprendizaje, desarrollan cátedras relacionadas al manejo de pacientes en estado crítico o con alto riesgo de mortalidad, según el "Plan Curricular de la Carrera de Enfermería", del año 2014 (Bonilla, 2014, p. 1). En este contexto, tercer semestre contiene el programa "Enfermería para el Cuidado Integral del Adulto y Adulto Mayor II", donde se abordan los problemas clínico quirúrgico de mayor complejidad según el perfil epidemiológico de la realidad del país; sexto semestre incluye el programa "Enfermería avanzada", que proporciona cuidados integrales de enfermería al paciente en estado crítico, en octavo semestre realizan prácticas pre-profesionales en los servicios, abordando módulos relacionados al cuidado integral del neonato y emergencias pediátricas, en centro obstétrico y quirúrgico (Nuñez, 2017, p. 5). Los estudiantes del Internado Rotativo estuvieron expuestos porque estaban finalizando su carrera y tenían que aprobar múltiples estancias que incluían la Unidad de titulación, las prácticas como tal y el examen de habilitación profesional vigente, para continuar con su año de 
trabajo en salud rural, las cuales, en conjunto, los exteriorizan a mayores presiones académicas.

En cuanto al análisis de las subescalas por semestre, las cifras son preocupantes, porque hay un $19,2 \%$ que tiene agotamiento, un $18,6 \%$ cinismo y el $29,7 \%$ de estudiantes con baja autoeficacia académica. Estos resultados difieren de manera estadísticamente significativa con la investigación realizada por Felipa (2015), denominada "síndrome de burnout en Internos de Enfermería de la Universidad Privada Arzobispo Loayza", donde se halló alto nivel de: agotamiento emocional en un $53,2 \%$ de estudiantes y de despersonalización en el $40,3 \%$, además un bajo nivel de realización personal en el 55,8\%.

En lo referente a los datos sociodemográficos, todos los estudiantes con síndrome de burnout académico corresponden al sexo femenino y tienen estado civil soltero. Por otro lado, la mitad de ellos, viven solos o con familiares con lazos de consanguinidad lejanos. En este contexto, de los datos obtenidos en comparación con un estudio realizado por Rísquez, García, y Sabuco (2012), concuerdan con que éste Sindrome predomina en el sexo femenino con un $75,7 \%$ de la muestra y además el $57,8 \%$ tienen estado civil soltero. Por otro lado estos datos coinciden con Bolaños y Rodriguez (2015), quienes concluyeron que la presencia de este síndrome puede estar relacionado con el sexo femenino, porque las mujeres tienen mayor incidencia de sufrir trastornos interiorizantes, tales como depresión, falta de apoyo familiar, mayores presiones ante exámenes, entre otros.

En cuanto a uno de los factores personales más importantes asociados al síndrome de burnout académico, un gran número de los encuestados manifestó dormir menos de 6 horas al día, de ellos el 1,7\% tiene este síndrome. Estos resultados difieren mucho en comparación a un estudio sobre "síndrome de burnout, estrés laboral y calidad de vida en trabajadores de enfermería", donde obtuvieron que la población duerme alrededor de 7 horas al día, lo cual está dentro del rango recomendado (Vidotti, Trevisan, Quina, Perfeito, y Cruz, 2019, p. 348). Estos datos señalan que dormir menos de 6 horas al día puede ser perjudicial para la salud, provocar los niveles altos de agotamiento y las diversas manifestaciones encontradas, también éste déficit podría deberse a la mala planificación o utilización del tiempo, por ejemplo, relacionado con el uso excesivo del celular y/o computador. Desde esta perspectiva la "National Sleep Foundation's Sleep Time Duration Recommendations" (Hirshkowitz, y otros, 2015, p. 1), señala que las horas de sueño recomendadas para personas entre 18 a 64 años son de 7 a 9 horas con un mínimo de 6 y un máximo de 10 a 11 horas.

Desde el enfoque de los factores académicos, el 59\% indicó que esta carrera no fue la primera opción, durante la postulación en el Sistema de Educación Superior, utilizado en el momento de su ingreso, pudiendo ser un factor potencial sumado a resultados en evaluaciones cognitivas con un mayor riesgo de deserción académica y a su vez se asocia con una inadecuada orientación educativa y profesional al elegir la carrera (Marenco et al, 2017, p. 47) (DCI-ALA, 2012, p. 1). Estos resultados concuerdan con Cuevas (2013, p. 83) donde también concluyeron que, a mayor nivel de estrés, menor rendimiento académico, lo cual puede desencadenar deserción estudiantil.

Cabe mencionar que más del $90 \%$ de alumnos manifestó que, si recibe tutorías académicas tanto en clases como en práctica, sin embargo, de ellos el 1,7\% tiene síndrome de burnout académico, lo que se contradice con la bibliografía revisada (Ticona et al, 2010, p. 5). Por otro lado, todos los estudiantes que tienen síndrome de 
burnout académico prefieren realizar trabajos individuales, resultado que concuerda con Trujillo (2017), al ser considerado como uno de los factores asociados.

El $41 \%$ de estudiantes indica que a veces tiene dificultad en el acceso a las bibliotecas de la carrera tanto físicas como virtuales, lo que concuerda con la revisión bibliográfica citada (Ticona et al, 2010, p. 5). Por otro lado el $60 \%$ de alumnos indicó que nunca han tenido acceso a la asociación estudiantil de la Carrera y dentro de este grupo están los estudiantes que tienen burnout académico, cabe recalcar que a pesar de existir dicha asociación en la carrera, es evidente que la misma no favorece la apertura hacia los estudiantes para lograr una interacción con ellos, lo que puede ser un factor estresante que deteriora el bienestar estudiantil, según lo afirma (Norabuena, 2016, p. 18).

Por otro lado, el $68 \%$ de los estudiantes consideran que solo a veces la metodología de enseñanza en la teoría-práctica es adecuada, lo cual puede ser un factor estresante, según lo indican Estrada et al. (2017), por ello es necesario que los docentes evalúen de manera constante éste aspecto y apliquen un mejoramiento continuo. En lo que se refiere a la satisfacción con las temáticas abordadas, todos los estudiantes con el síndrome, manifestaron estar satisfechos, lo cual a su vez refleja su pertinencia acorde a la misión y visión de la Institución, pero es contradictorio con lo que afirma Neave (2016), en su trabajo sobre el impacto del estrés y burnout, en la salud; Así mismo, los autores Urrejola, G, Vásquez, C, Tiscornia, C, Lisperguer, S, y Calvo, S (2019), afirman según los resultados obtenidos que no hay una relación directa entre la metodología de enseñanza y el rendimiento académico. No obstante, la experiencia académica previa parece ser de gran importancia y aporta de manera significativa al sistema de educación superior, por ello es necesario que los estudiantes expresen sus necesidades educativas a sus maestros, de acuerdo con la realidad sanitaria encontrad durante las prácticas en las Unidades Asistenciales Docentes.

Por otro lado, el $40 \%$ de universitarios mencionó que casi siempre domina las habilidades y destrezas, lo cual es positivo para el grupo de profesionales, sin embargo, los estudiantes que tienen Bunout académico están dentro de este grupo, por lo que estos resultados se contradicen con la afirmación de Rísquez et al, (2012), donde menciona que la deficiencia en éste aspecto puede causar síndrome de burnout. En este sentido y considerando que la Carrera tiene dentro de su plan curricular el acceso a las prácticas preprofesionales, no solo en las UAD, sino también en la clínica de simulación y es probable que este beneficio disminuya significativamente el estrés que implica realizar ciertos procedimientos en el paciente real sin antes haberlo aplicado a un paciente que actúa como simulador de escenarios, incluso cabe mencionar que ésta hipótesis tenga relación con la baja prevalencia de síndrome de burnout académico encontrada.

Caballero, Breso y González (2015), mencionan que la competitividad entre compañeros es uno de los factores más destacados para el desarrollo del síndrome de burnout, en este sentido concuerdan los resultados encontrados, puesto que se determinó que el 35\% de estudiantes sienten que en el grupo académico siempre existe competitividad. Por otra parte, el $78 \%$ de estudiantes expresaron que nunca ha recibido alguna beca económica por excelencia académica. En este contexto tanto la competitividad, como la falta de incentivos económicos, son considerados como factores académicos estresantes según lo indican otras investigaciones realizadas por (Estrada et al, 2017, p. 2) y (Uribe y Illesca, 2016, p. 1). Se observa que $43 \%$ de 
estudiantes, indicaron que casi siempre o siempre tienen temor y ansiedad durante las evaluaciones académicas, resultados que afirman el estudio de Macbani et al, (2018).

Finalmente, en cuanto a los factores del contexto socioeconómico, el $76 \%$ de la muestra encuestada señaló que por lo general tan solo a veces o en otros casos nunca tienen deudas económicas, sin embargo, dentro de este grupo está el 2,3\% de estudiantes que tienen síndrome de burnout académico, en esta ocasión los resultados encontrados coinciden con Norabuena (2016). Se ha observado que muchos de los estudiantes acuden a la Universidad con escasos recursos económicos esto genera mayor impacto negativo en el ámbito del estrés cuando ellos/ellas residen de manera temporal en la ciudad, además implica un gasto adicional en arrendar una vivienda y en múltiples ocasiones no logran cubrir su alimentación, alquiler del servicio de telefonía, internet, impresiones, transporte, vestimenta, entre otros. Adicionalmente, es interesante recordar que el factor económico es un determinante de estrés en la generación actual, puede estar relacionado con el proyecto de vida que no está bien definido en la mayoría de las jóvenes y en ocasiones los recursos económicos son malgastados en ciertas adicciones o pasatiempos que no son saludables.

Desde el enfoque del burnout laboral, en la muestra estudiada correspondiente a estudiantes del Internado Rotativo, el $4 \%$ de alumnos de séptimo y octavo semestre respectivamente, tienen este síndrome, con una probabilidad de 1,22\%. En cuanto a las subescalas, de forma agrupada con el semestre, niveles altos de cansancio emocional se encontró en iguales porcentajes 30\% de estudiantes, tanto en séptimo como en octavo semestre, mientras que, en cuanto a los niveles altos de despersonalización, el porcentaje es más relevante en séptimo $(10 \%)$ y finalmente en lo que se refiere a niveles bajos de realización personal se observa también en séptimo (12\%). Estos resultados concuerdan con algunos autores, entre ellos están (Acurio y Córdova, 2015, p. 1), quienes encontraron según los criterios del MBI, que las Enfermeras presentan agotamiento emocional en un 16,3\%; despersonalización $16,3 \%$; baja realización personal $37,2 \%$, lo que aumenta el riesgo para presentar el síndrome de burnout.

En lo referente a los factores laborales más estudiados, la mitad de los universitarios con síndrome de burnout laboral tiene exceso de horas, lo cual podría estar relacionado con el cansancio, según la afirmación de (Marenco et al, 2017, p. 2) y otros autores investigados (Díaz, 2017, p. 2) (Acurio y Córdova, 2015, p. 3) (Cabrera, 2017, p. 4) (Nuñez, 2017, p. 6). Actualmente, este problema ya fue solucionado, porque los estudiantes realizan únicamente las horas dispuestas por el reglamento en todas las Unidades de rotación.

El grupo de estudiantes que tienen burnout laboral, refieren que algunos médicos, enfermeras/os $u$ otro personal de salud se dirigen hacia ellos en tono autoritario, algunos han tenido contacto con el dolor y riesgo inminente de mortalidad. Finalmente, el $4 \%$ de internos/as indicaron haber manejado pacientes en estado crítico o de alta complejidad. Estos resultados son afirmados por (Gavilema y Pilamunga, 2014, p. 9) y (Moscoso y Delgado, 2015, p. 6). También (Acurio y Córdova, 2015 , p. 5), evidencian la presencia del síndrome de burnout en un $9 \%$ de enfermeras especialistas en medicina crítica, mismas que tienen conocimiento acerca del manejo del paciente crítico, por lo que estas áreas pueden generar estrés y por ende repercutir en el rendimiento, capacidad laboral, y atención al usuario. 
En lo que se refiere a las manifestaciones asociadas (adicionales a las valoradas en las subescalas), llama la atención que se evidencien valores estadísticamente significativos en éstas; más del $50 \%$ de estudiantes han presentado nausea, gastritis, gripe frecuente, rigidez muscular, dolor de cabeza, de cuello y de espalda, por lo que es importante socializar con los estudiantes sobre la relación de dichos síntomas con el riesgo de presentar síndrome de burnout, según lo citado por (Flores, 2016, p. 45).

En el ámbito emocional, los resultados afirman que la población estudiada tiene alto riesgo desarrollar síndrome de burnout laboral, según (Estrada et al, 2017, p. 1) y (Marenco et al, 2017, p. 47). Se observan muy altos porcentajes de alteraciones, por ejemplo, el $89 \%$ refirió que ha tenido dificultad para la concentración, un $82,6 \%$ cambios bruscos de humor, seguido de ansiedad el $79,1 \%$, soledad un $64,5 \%$ y baja autoestima el 55,2\%. Por lo que es importante considerar estas cifras con las autoridades de la institucion y tomar medidas tanto correctivas como preventivas.

Las manifestaciones conductuales, señalan que un alto porcentaje de alumnos $59,9 \%$ consumen alcohol, pero también existe evidencia sobre el consumo de tabaco $20,3 \%$, de fármacos $13,4 \%$ y de drogas $5,2 \%$. Lamentablemente, el $18,6 \%$ de la muestra indicó que han tenido pensamientos o intentos de suicidio, por lo que una vez más se demuestra la canalización inadecuada del estrés como lo afirman varios autores en sus investigaciones (Benalcazar, 2017, pág. 1) (Mesías, 2016, pág. 3) (Granda, 2016, pág. 2) (TN, 2017, p. 4). Adicionalmente, los resultados son similares a los encontrados por García, Vega, Farías, Améstica, y Aburto (2020) donde el $29,8 \%$ consume alcohol los fines de semana, y en contraste el consumo de tabaco es una de las variables que explica el alto cansancio emocional.

El $55,8 \%$ ha pensado en abandonar la carrera; respecto a la deserción académica, si ésta se produce a nivel superior existen varios motivos que provocan la no permanencia en la universidad, según lo señala Sinchi (2018). Estos resultados están acorde a la Guía de Gestión Universitaria Integral del Abandono (2012), que indica que los factores más influyentes en la deserción de abandono incluyen problemas económicos que influyen directamente sobre el desarrollo de sus estudios, dificultad para compatibilizar estudio y trabajo, inadecuada orientación educativa y profesional al elegir la carrera, problemas personales o familiares y falta de motivación personal por los estudios. Por otro lado, en una publicación realizada por Diario "EI Telégrafo" en el año 2016, señala que "El 26\% de los alumnos universitarios abandonó sus estudios hace dos años en los centros públicos y privados del país, según la Secretaría de Educación Superior, Ciencia, Tecnología e Innovación (SENESCYT)", mientras que la Organización de las Naciones Unidas para la Educación, la Ciencia y la Cultura (UNESCO), indica que la tasa de abandono llega a ser aproximadamente del 40\% a este nivel de formación (Acosta, 2019, p. 11).

Es relevante mencionar que se encontró 37 correlaciones entre variables correspondientes a los factores asociados al síndrome de burnout y manifestaciones, con un valor de chi cuadrado $<0,05$, sin embargo todas ellas demuestran una dirección y fuerza de la relación estadísticamente baja con un coeficiente de contingencia $<0,30$. A su vez, dentro de las 37 variables, solo 4 correlaciones, mantienen una $p$ de 0,00 y un coeficiente de contingencia entre 0,27 y 0,29. En éstas se encontraron, que la mayoría de los estudiantes que no pueden controlar sus situaciones de estrés, a la vez tienen ansiedad y baja autoestima. También se halló que los estudiantes que sienten temor durante sus evaluaciones académicas, a la vez tienen sentimientos de soledad. Finalmente, gran parte de los universitarios que no 
tienen actividades de ocio y/o relajación tienen baja autoestima. Estos resultados concuerdan con lo citado por varios autores entre ellos (Gavilema y Pilamunga, 2014, p. 5) y (Macbani et al, 2018, p. 3), quienes afirman la relación entre dichas variables.

En cuanto a las limitaciones potenciales que existieron durante el desarrollo del estudio, fue en la aplicación del instrumento de evaluación a los distintos semestres que formaron parte de la muestra, ya que fue complicado coordinar entre el tiempo de las investigadoras y de la población investigada, por la diferencia de carga horaria, responsabilidades y variabilidad de los días en los que cumplen con las actividades teóricas como prácticas en cada semestre.

\section{CONCLUSIONES}

La prevalencia del síndrome de burnout encontrada fue baja, en este contexto en el de tipo académico fue de $2,3 \%$, con una probabilidad de $3,52 \%$. En las subescalas hay altos niveles de agotamiento en un 19,2\%, cinismo $18,6 \%$ y $29,7 \%$ tuvo autoeficacia académica baja. Por otro lado, en el de tipo laboral, en estudiantes del Internado Rotativo, fue de $4 \%$, con una probabilidad de $1,22 \%$. Subescalas: cansancio emocional $60 \%$, despersonalización $12 \%$ y baja realización personal $18 \%$.

Dentro de las características sociodemográficas, se determinó que predomina el sexo femenino, por lo que hay una mayor vulnerabilidad a desarrollar este síndrome, debido a que existe una múltiple carga emocional en el ámbito académico, social y familiar, considerando que el $25 \%$ de la muestra tiene cargas familiares, lo cual es un agravante para incrementar el nivel de estrés.

En cuanto a los factores personales asociados al síndrome de burnout académico, el $68 \%$ de estudiantes duerme $<6$ horas al día, lo que es una problemática en el desempeño de sus actividades. En el ámbito académico, el $28 \%$ consideró que la metodología de enseñanza en la teoría-práctica a veces es adecuada y el $35 \%$ que siempre existe competitividad entre compañeros, el 78\% nunca ha recibido una beca económica por excelencia académica y el $40 \%$ a veces dominó las habilidades y destrezas. De quienes tienen el burnout laboral, la mitad de internos tiene exceso de horas, indicó que algunos médicos, enfermeras/os u otro personal de salud se han dirigido hacia ellos en tono autoritario, ha tenido contacto con el dolor y riesgo inminente de mortalidad y algunos de ellos han manejado pacientes en estado crítico o de alta complejidad.

Las principales manifestaciones asociadas al síndrome de burnout, que presentaron los estudiantes fueron: entre las físicas; más del 50\%, náusea, palpitaciones, gastritis, gripe frecuente, rigidez muscular, dolor de cabeza, cuello y espalda. Las manifestaciones emocionales; más del 55\%, incluyeron dificultad para la concentración, cambios bruscos de humor, ansiedad, soledad y baja autoestima y las conductuales; consumo de alcohol $55,8 \%$ y pensamientos sobre abandonar la carrera $59,9 \%$. Todos los resultados en conjunto hacen necesaria una intervención para tratar y prevenir casos de burnout académico en mayor proporción que I aboral.

\section{BIBLIOGRAFÍA}

Acosta, K. (2019). Recuperado el 10 de 2016, de La deserción universitaria bordea el 40\%. El Telégrafo: https://www.eltelegrafo.com.ec/noticias/ sociedad/4/la-desercionuniversitaria-bordea-el-40 
Acurio, H., y Córdova, M. (2015). Valoración del síndrome de burnout en la salud de las Enfermeras de Cuidados Intensivos del Hospital Eugenio Espejo, 2014. Quito: Instituo de Postgrado.

Aguas, A., Vargas, L., Guzman, M., y Ferrer, M. (2017). Recuperado el 03 de 06 de 2018, de Presencia del Síndrome de Burnout en estudiantes Odontología y optometría de la Universidad Santo Tomás en el año 2015.: http://repository.usta.edu.co/bitstream/handle/11634/4770/AguasRozoAngelicaVarga sCalderonLauraGuzmanMillanMillerFerrerCristanchoMichelle2017. pdf?sequence $=1 \mathrm{yi}$ sAllowed $=y$

Benalcazar, J. (2017). Recuperado el 13 de mayo de 2018, de Síndrome de Burnout, en el personal de enfermería del primer nivel de atención en salud Distrito 10D01 Imbabura 2017: http://repositorio.utn.edu.ec/handle/123456789/7352

Bolaños, N., y Rodriguez, N. (2015). Prevalencia del Síndrome de Burnout académico en el estudiantado de Enfermería de la Universidad de Costa Rica. Sistema de Información Científica Redalyc- Red de Revistas Científicas de América Latina y el Caribe, España y Portugal, 1. Recuperado el 15 de julio de 2018, de Prevalencia del Síndrome de Burnout Académico en los y las estudiantes de la Licenciatura en Enfermería.: https://www.redalyc.org/jatsRepo/448/44846315002/html/index.html

Bonilla, J. (2014). Plan Curricular de la Carrera de Enfermería. Quito: UCE.

Bresó, E., Salanova, S., Schaufeli, W., Equipo de investigación, W., y Nogareda, C. (2013). Centro Nacional de "Condiciones de trabajo". Recuperado el 3 de junio de 2018, de NTP 732: Síndrome de estar quemado por el trabajo "Burnout" (III): Instrumento de Medición: https://www.insst.es/documents/94886/327446/ntp_732.pdf/bf45e6442986-42b0-b9a5-ce5bef2917bd

Caballero, C., Breso, E., y González, O. (2015). Burnout en estudiantes universitarios. Psicología desde el caribe, 32(3), 434.

Cabrera, E. (2017). Recuperado el 16 de julio de 2018, de Syllabus de Enfermería para el Cuidado Integral del Adulto y Adulto Mayor II: http://akacdn.uce.edu.ec/ares/w/facs/fcm/CarreraEnfermeria/Syllabus/Syllabus\%201717/301\%20ENFERMERIA\%20PARA\%20EL\%20CUIDADO\%20INT\%20DEL\%20AD \%20Y\%20AD\%20MAYOR\%20I\%202017-2017.pdf

Cuevas, A. (2013). Influencia del Burnout académico en la probabilidad de deserción en estudiantes universitarios. Bogotá: Universidad Piloto de Colombia.

DCI-ALA. (2012). Gestión Universitaria Integral del Abandono. Recuperado el 12 de 2018, de Proyecto ALFA GUIA DCl-ALA: http://www.alfaguia.org/wwwalfa/images/resultados/InformeEncuestaOpinion2012.pdf

Díaz, A. (2017). Recuperado el 03 de 06 de 2018, de "VI Jornada Estatal de Salud. Prevención y seguridad en el trabajo". Síndrome de Burnout.: http://secadva.unach.mx/images/PERSONAL/PDF/sexta/Sindrome_Burnout._CEA.p $\mathrm{df}$

Estrada, H. H., De La Cruz, A. S., Bahamón, M. J., Perez, J. M., y Caceres, A. M. (2017). Academic burnout and its connection with psychological wellbeing in college students. Espacios, 39(15), 9. Recuperado el 26 de Mayo de 2018, de Academic burnout and its connection with psychological wellbeing in college students: http://www.revistaespacios.com/a18v39n15/a18v39n15p07.pdf

Felipa, A. (2015). Síndrome de Burnout en internos de enfermería de la Universidad Privada Arzobispo Loayza. Lima-Perú: Universidad Privada Arzobispo Loayza.

Flores, E. (2016). Burnout en los Internos Rotativos de Medicina del Hospital José María Velasco Ibarra, enero 2016- mayo 2016 (1 ed.). (T. d. Grado, Ed.) Ambato: UNIANDES.

FORBES. (2017). Recuperado el 10 de 2019, de mexicanos, los más estresados del mundo por su trabajo-: https://www.forbes.com.mx/mexicanos-los-mas-estresados-delmundo-por-su-trabajo/ 
García, V., Vega, Y., Farías, B., Améstica, L., y Aburto, R. (2020). Factores Asociados al Burnout Académico en Estudiantes de Internado Profesional de Fonoaudiología. Ciencia y Trabajo (62).

Gavilema, E., y Pilamunga, M. (2014). Factores de riesgo asociados al Síndrome de Burnout en el personal profesional de Enfermería del Hospital Dr. Roberto Gilbert Elizalde, provincia del Guayas. Mayo 2013 - marzo 2014. Guaranda - Ecuador: UNIVERSIDAD ESTATAL DE BOLÍVAR.

Granda, D. (2016). Recuperado el 15 de julio de 2018, de Las dimensiones del síndrome de burnout académico (agotamiento, cinismo o eficacia profesional) que se presenta con mayor prevalencia en un grupo de 90 estudiantes de psicología de la Corporación Universitaria Minuto de Dios Seccional Bello: http://repository.uniminuto.edu:8080/xmlui/bitstream/handle/10656/5168/TP_Granada BorjaDaniela_2016.pdf?sequence $=1$

Hirshkowitz, M., Whiton, K., Albert, S. M., Alessi, C., Bruni, O., Carlos, L., . . . Adams, H. (2015). National Sleep Foundation's sleep time duration recommendations: methodology and results summary. Sleep Health, 1(1):40-3.

Jiménez, Á., y García, S. (2016). Valoración de ansiedad y burnout en los profesionales de enfermería de un servicio de Nefrología. Enfermería Nefrológica-Scielo, 19(2), 119.

Macbani, P., Ruvalcaba, J. C., Vásquez, P., Ramírez, A., González, K., Arredondo, K., . . . Trejo, A. (2018). Estrés académico, estresores y afrontamiento en estudiantes de Odontología en el Centro de Estudios Universitarios Metropolitano Hidalgo [CEUMH]. Journal of Negative and No Positive Results (9), 465, 558.

Marenco, A., Suarez, Y., y Palacio, J. (Julio - diciembre de 2017). Burnout académico y síntomas relacionados con problemas de salud mental en universitarios colombianos. Psychol, 11(1), 46. Recuperado el 26 de mayo de 2018, de Burnout académico y síntomas relacionados con problemas de salud mental en universitarios colombianos: http://www.scielo.org.co/pdf/psych/v11n2/1900-2386-psych-11-02-00051.pdf

Martínez, C. H., y Caballero, C. C. (2016). Sistema de Información Científica Redalyc ${ }^{\circledR}$. Recuperado el 26 de mayo de 2018, de Validación del cuestionario Maslach Burnout Inventory-Student Survey (MBI-SS) en contexto académico colombiano. Revista CES Psicología, 9(1), xx-xx.: http://www.redalyc.org/articulo.oa?id=423545768002

Mendizábal, S., Noboa, S., Cueva, G., y Buitrón, R. (2017). Bioestadística. 1a ed. En Bioestadística. 1a ed. (pág. 168). Quito: L. 017.

Mesías, A. R. (2016). "Plan de prevención del síndrome de burnout y su influencia en la satisfacción del usuario del servicio de consulta externa de una institución de salud pública." (1 ed.). (E. d. PUCE, Ed.) Ambato: PUCE.

Moscoso, M. (2014). Recuperado el 3 de junio de 2018, de El estudio científico del estrés crónico en neurociencias y psicooncología: http://www.redalyc.org/pdf/1471/147137147003.pdf

Moscoso, M., y Delgado, E. (junio de 2015). The theory of chronic stress as a scientific model in Cognitive Neuroscience. Revista IIPSI, 18 (N.․ 1 - 2015).

Neave, R. (2016). Recuperado el 03 de 06 de 2018, de Estrés laboral, Burnout y su impacto en la salud. Psiquiatría General C.E.D.H.N.L: http://www.cedhnl.org.mx/imagenes/publicaciones/presentaciones/CEDHNL_VIISemi narioDHS/ModuloIV/Estres-Laboral-y-burnout-CEDH.pdf

Norabuena, A. (2016). Recuperado el 10 de julio de 2018, de Nivel de Síndrome de Burnout en estudiantes de enfermería de la segunda especialidad de Enfermería en Centro Quirúrgico de la Universidad Nacional Mayor de San Marcos. Lima:: http://cybertesis.unmsm.edu.pe/bitstream/handle/cybertesis/6279/Norabuena_gm.pdf ? sequence $=1$ yisAllowed $=y$

Nuñez, A. (2017). Syllabus Enfermería avanzada Enfermería. Quito: UCE.

Ramírez, M. (2017). Recuperado el 13 de mayo de 2018, de El estrés no afecta solo a la parte emocional, sino que también se presenta como un cuadro de alteración física: 
http://www.revistagestion.ec/sites/default/files/2017-

08/UTPL_PERSPECTIVAS_PDF.pdf

Rísquez, M. I., García, C. C., y Sabuco, E. D. (2012). Resilience and Burnout syndrome in nursing students and its relationship with sociodemographic variables and interpersonal relationship. Int J Psychol Res, 5(1)(88), 88-95.

Rosales, R. (2014). Recuperado el 3 de junio de 2018, de Estrés y Salud: http://tauja.ujaen.es/bitstream/10953.1/952/1/TFG_RosalesFern\%C3\%A1ndez\%2CR osaura.pdf

Sinchi, E. (junio de 2018). Acceso y deserción en las universidades. Alternativas de financiamiento (13(2):274-87).

Ticona, S., Paucar, G., y Llerena, G. (2010). Recuperado el 26 de mayo de 2018, de Nivel de estrés y estrategias de aprontamiento en estudiantes de la facultad de enfermería UNSA Arequipa.: $\quad$ http://scielo.isciii.es/scielo.php?pid=S169561412010000200007yscript=sci_arttextytlng=en

TN. (2017). Recuperado el 21 de julio de 2018, de Síndrome de Burnout: más común entre profesionales que trabajan "las 24 horas": https://tn.com.ar/salud/lo-ultimo/sindromede-burnout-mas-comun-entre-profesionales-que-trabajan-las-24-horas_781687

Torres, M. (2017). Recuperado el 13 de mayo de 2018, de Prevalencia y factores de riesgo del Síndrome de Burnout, en el personal que labora en el Hospital de Solca de la ciudad de Loja: http://dspace.unl.edu.ec/jspui/bitstream/123456789/19654/1/TESISSOLCA-MARIA\%20TORRES.pdf

Trujillo, P. (2017). Correlación entre ansiedad, depresión, Burnout académico y desempeño en estudiantes: revisión bibliográfica [Tesis]. [Guayaquil-Ecuador]: Recuperado el 11 de 2018, de UNIVERSIDAD DE ESPECIALIDADES ESPÍRITU SANTO: ; 2017.http://repositorio.uees.edu

Uribe, M. A., y Illesca, P. M. (2016). Recuperado el 08 de Julio de 2018, de Burnout en estudiantes de enfermería de una universidad privada: http://www.redalyc.org/pdf/3497/349753310004.pdf

Urrejola, G, Vásquez, C, Tiscornia, C, Lisperguer, S, y Calvo, S. (2019). Estudio de la percepción sobre metodologías de enseñanza, experiencia académica previa y su relación con el rendimiento. Dialnet, 2.

Veletanga, J. (2017). Recuperado el 13 de 05 de 2018, de El burnout está presente en el 30\% de los sanitarios ecuatorianos: https://www.redaccionmedica.ec/secciones/profesionales/el-burnout-est-presente-enel-30-de-los-sanitarios-ecuatorianos-90910

Vidotti, V., Trevisan, J., Quina, M., Perfeito, R., y Cruz, M. (2019). Síndrome de burnout, estrés laboral y calidad de vida en trabajadores de enfermería. Enfermería Global. 